\title{
Murine plasma $N$-glycosylation traits associated with sex and strain
}

Karli R. Reiding ${ }^{1}$, Agnes L. Hipgrave Ederveen ${ }^{1}$, Yoann Rombouts ${ }^{1,2}$, Manfred Wuhrer*1

${ }^{1}$ Leiden University Medical Center, Center for Proteomics and Metabolomics, Leiden, The

Netherlands;

${ }^{2}$ Institut de Pharmacologie et de Biologie Structurale, Université de Toulouse, CNRS, UPS, France;

*Correspondence: Manfred Wuhrer, Leiden University Medical Center, Center for Proteomics and

Metabolomics, P.O. Box 9600, 2300 RC Leiden, The Netherlands; m.wuhrer@lumc.nl; Tel: +31-71-

$52-66396$ 


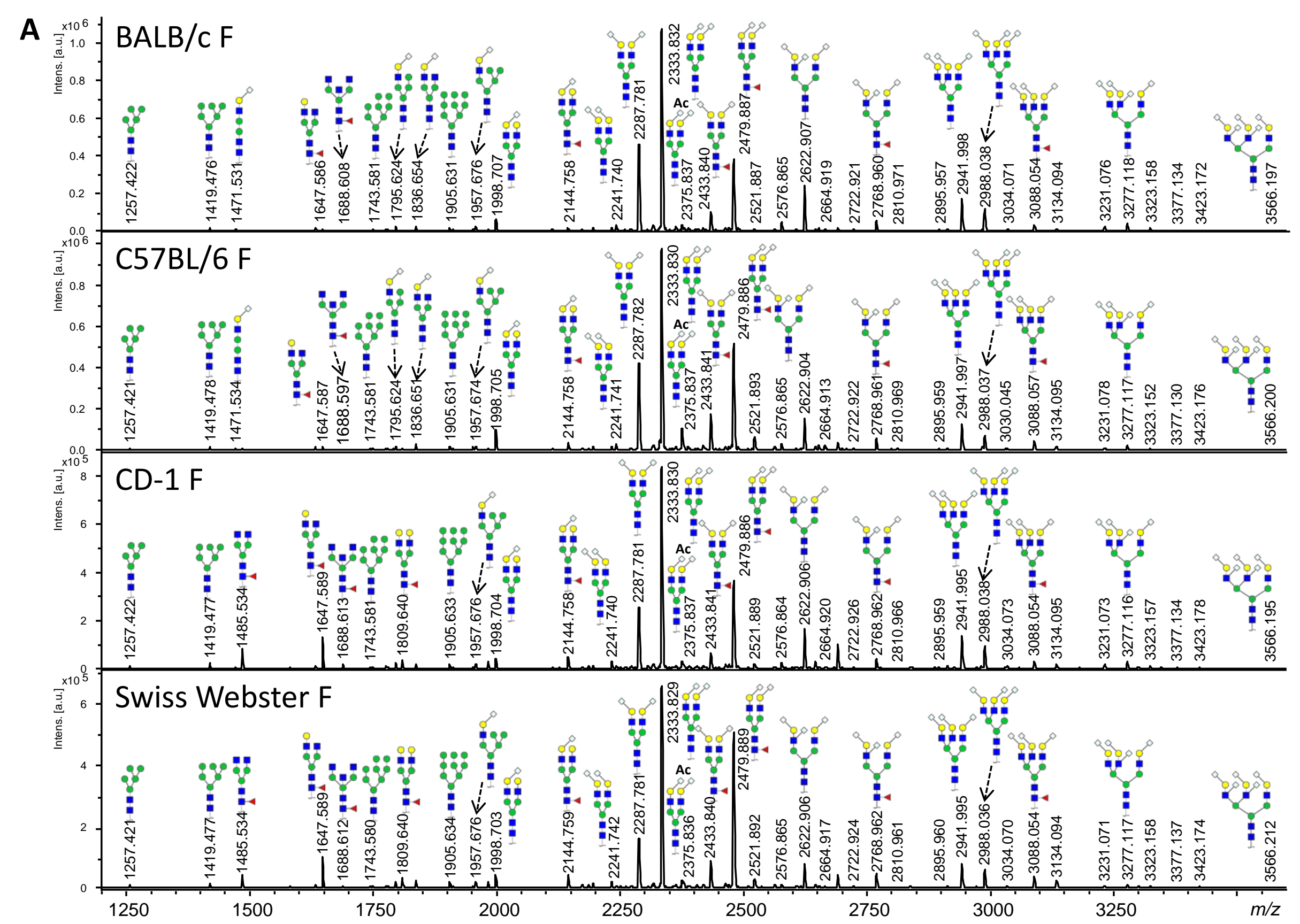

Figure S1. A) Female overall. Murine total plasma $\mathrm{N}$-glycome profile as measured by reflectron positive mode MALDI-TOF-MS after ethyl esterification. Glycan species are considered $[\mathrm{M}+\mathrm{Na}]^{+}$and assigned to signals on basis of recorded $\mathrm{m} / \mathrm{z}$ values and fragmentation. $\mathrm{N}$-Glycolylneuraminic acid linkages are determined by the method, as are the antennary compositions and fucose locations, whereas other linkages are presumed on basis of literature. 


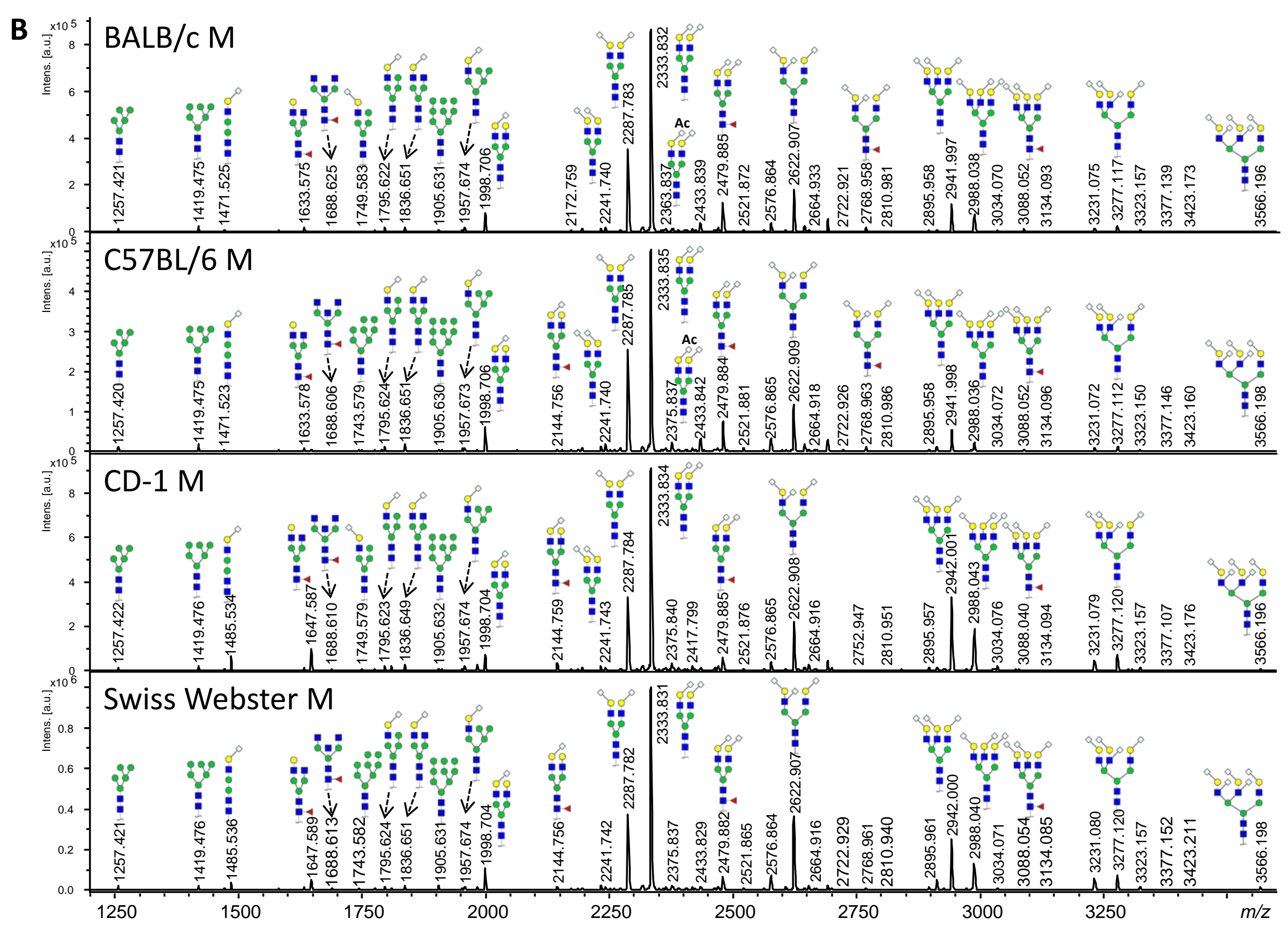

Figure S1. B) Male overall. Murine total plasma $\mathrm{N}$-glycome profile as measured by reflectron positive mode MALDI-TOF-MS after ethyl esterification. Glycan species are considered $[\mathrm{M}+\mathrm{Na}]^{+}$and assigned to signals on basis of recorded $\mathrm{m} / \mathrm{z}$ values and fragmentation. $\mathrm{N}$-Glycolylneuraminic acid linkages are determined by the method, as are the antennary compositions and fucose locations, whereas other linkages are presumed on basis of literature. 


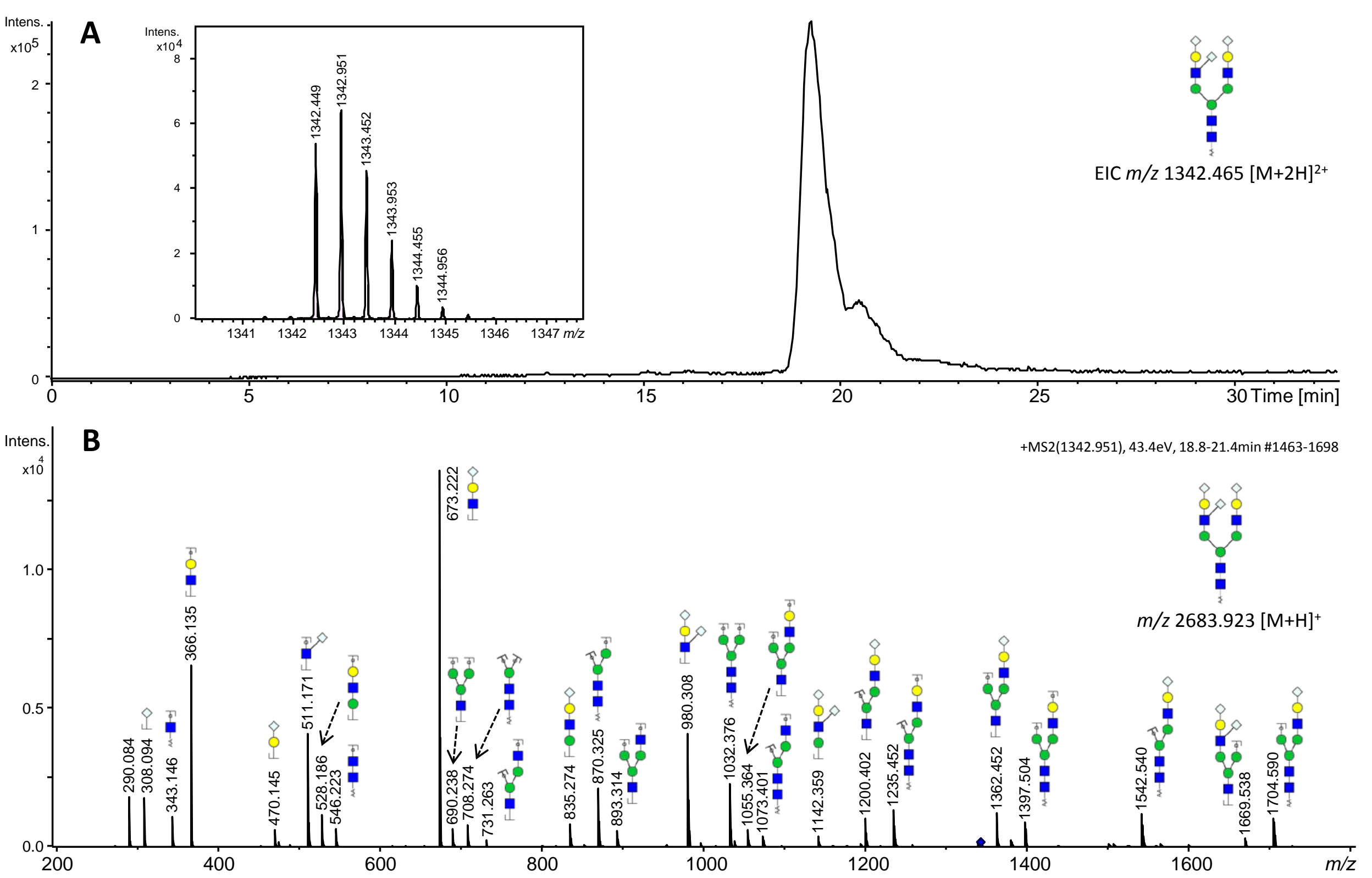

Supporting Figure S2. Reverse phase LC-ESI-QTOF-MS/MS analysis of CD-1 mouse mixed sex 2-aminobenzoic acid labeled plasma N-glycosylation. A) Extracted ion

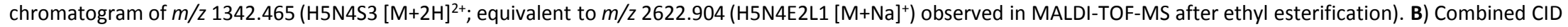

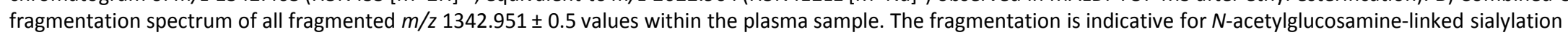

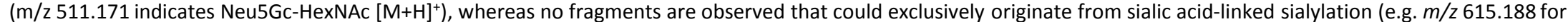
Neu5Gc-Neu5Gc $\left.[\mathrm{M}+\mathrm{H}]^{+}\right)$. 


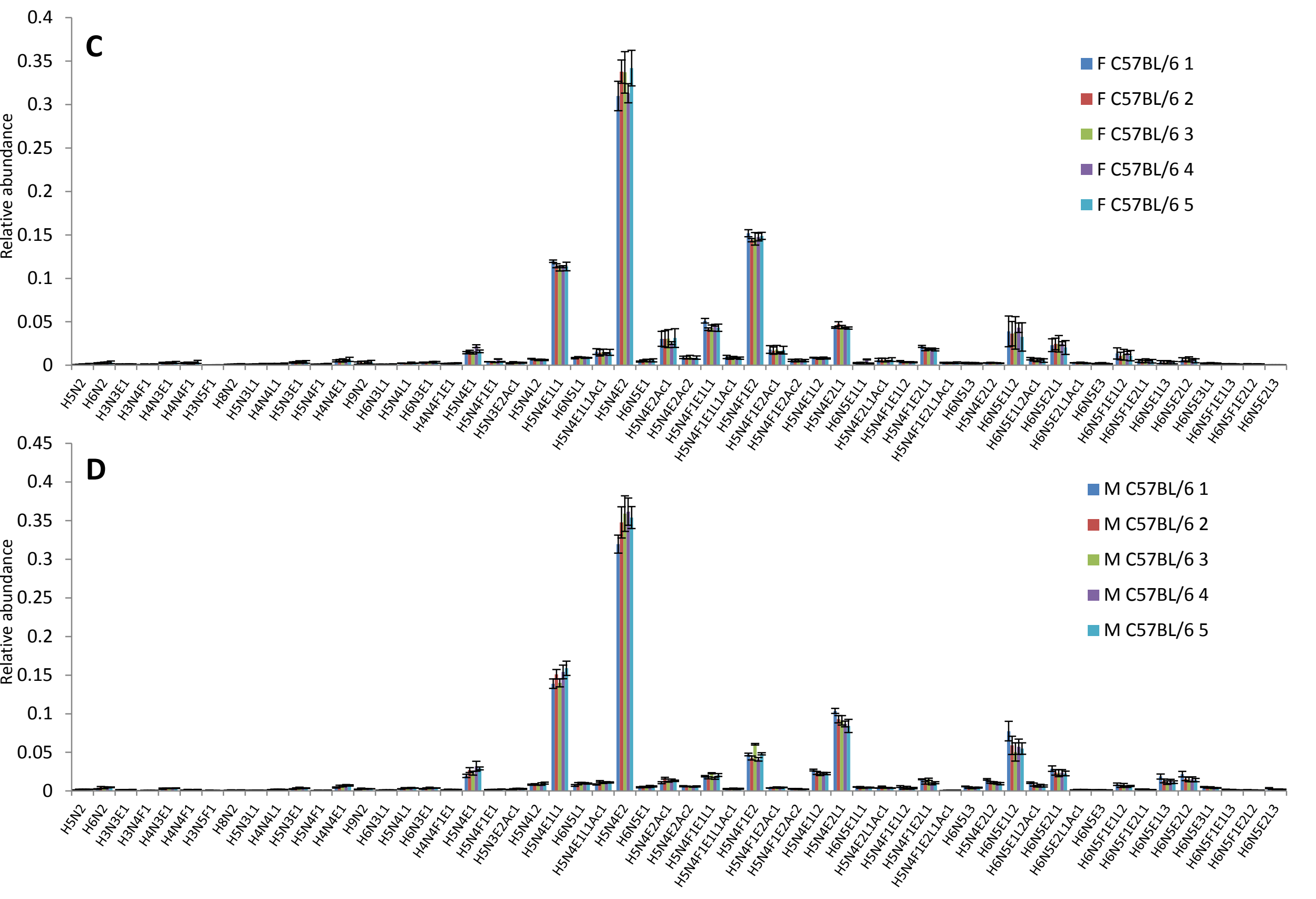

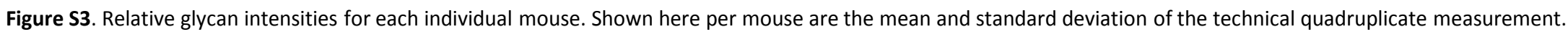
C) Female C57BL/6. D) Male C57BL/6. 


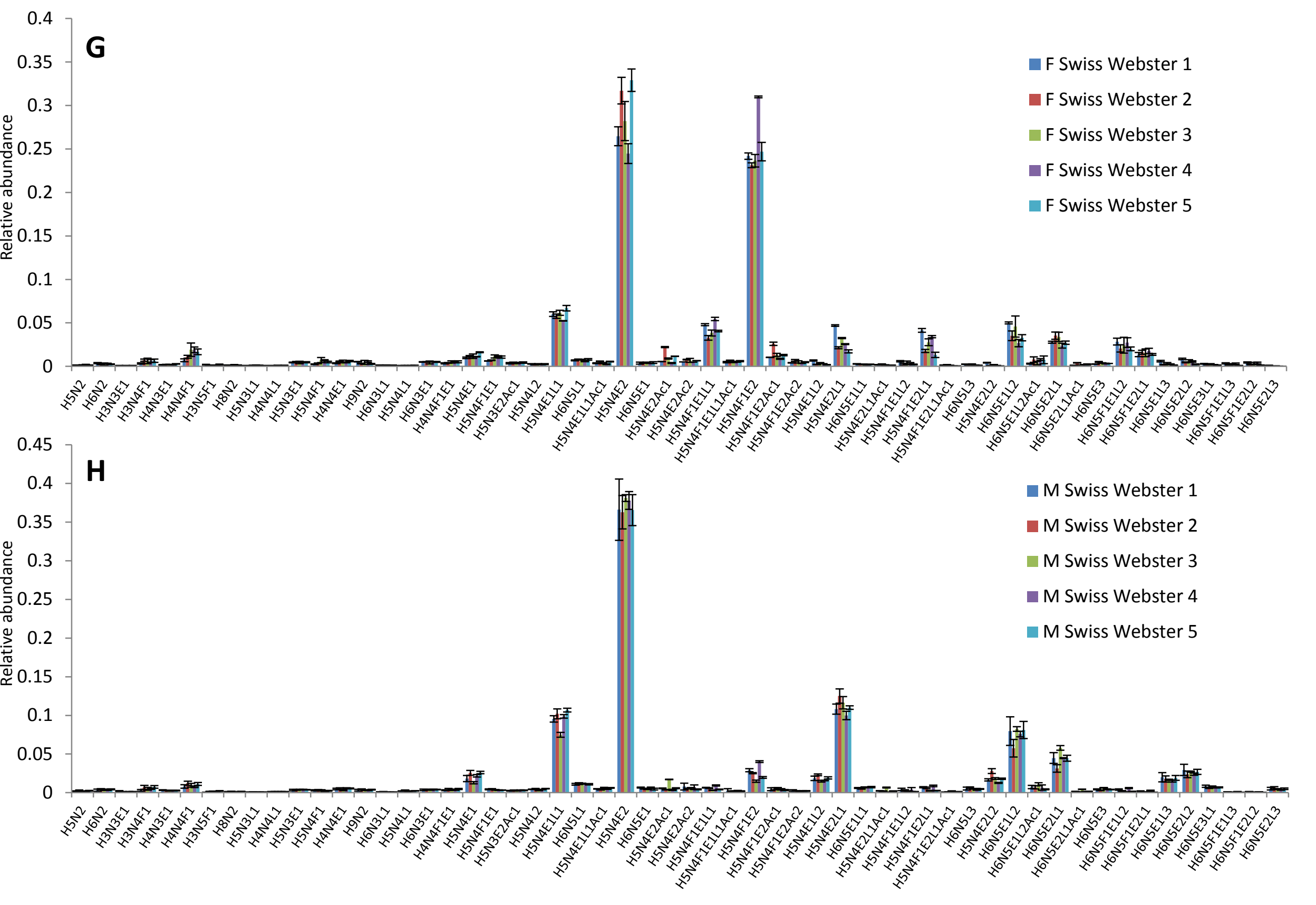

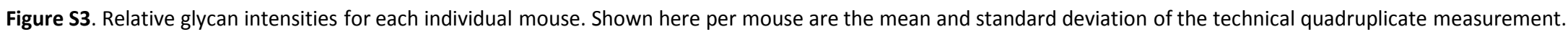
G) Female Swiss Webster. H) Male Swiss Webster. 


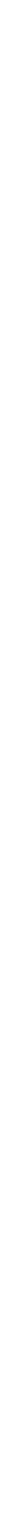

Figure S3. Mean and standard deviation of I) the male and female individuals from all strains, and J) the strains including both sexes. 

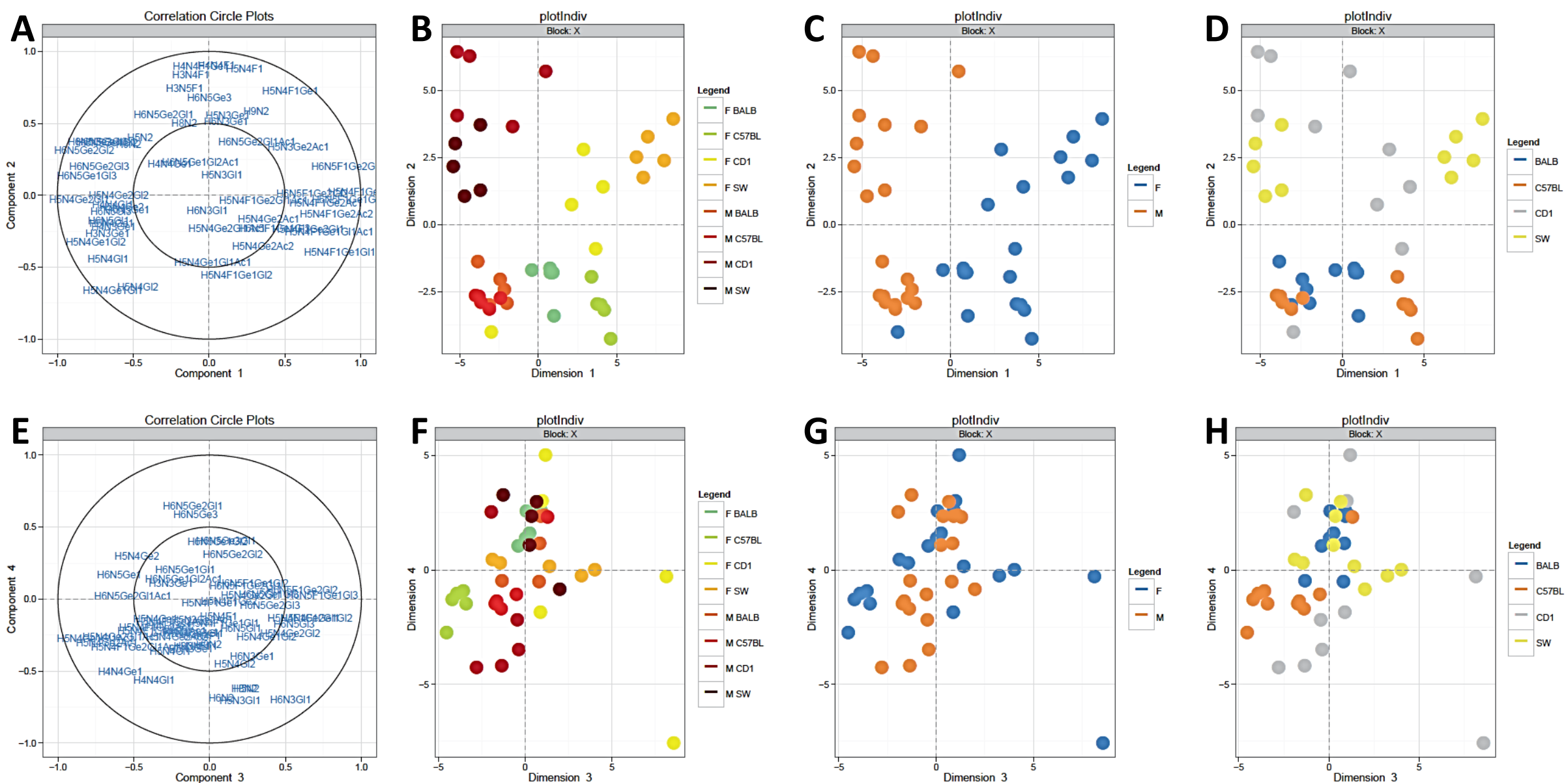

Figure S4. Principal component analysis of the glycan compositions calculated for the individual mice originating from a single strain and sex (averaged from up to four technical replicates). A) Loading plot of the first two principal components (PC1 and PC2), respectively explaining 31\% and $18 \%$ of the variance. B) Score plot of PC1 and PC2 colored by strain and sex. C) Score plot of PC1 and PC2 colored by sex. D) Score plot of PC1 and PC2 colored by strain. E) Loading plot of the third and fourth principal component (PC3 and PC4), respectively explaining $13 \%$ and $11 \%$ of the variance. F) Score plot of PC3 and PC4 colored by strain and sex. G) Score plot of PC3 and PC4 colored by sex. H) Score plot of PC3 and PC4 colored by strain. In general PC1 shows to largely separate the sexes, while PC2 separates strains BALB/C and C57BL/6 from strains CD-1 and Swiss Webster. The strains and sexes cluster well across the two components, but strains CD-1 and Swiss Webster show a larger individual variation than the BALB/C and $\mathrm{CD} 57 \mathrm{BL} / 6$ strains. For a further listing of the principal components see Table S6. 

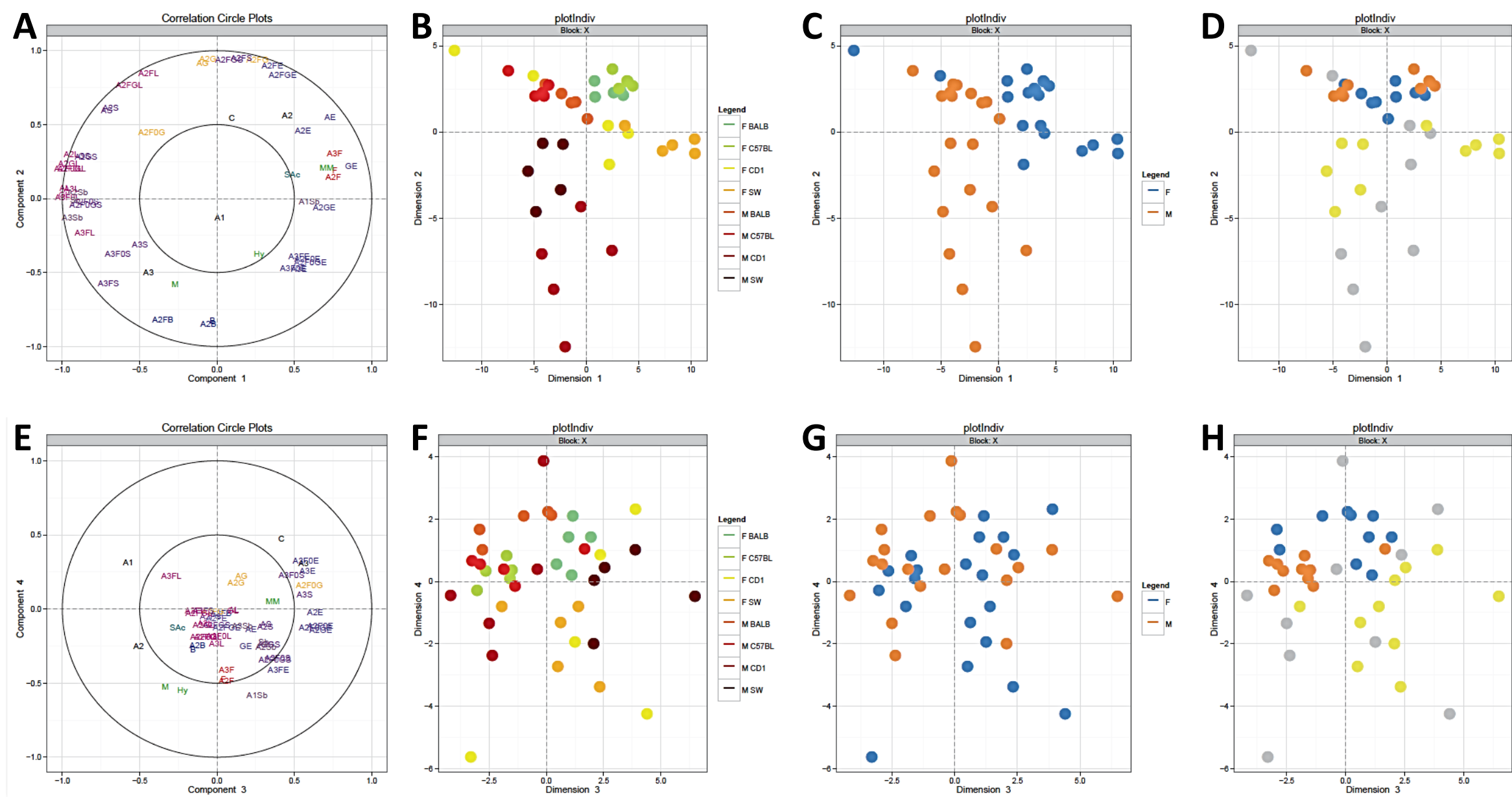

Figure S5. Principal component analysis of the derived glycan traits calculated for the individual mice originating from a single strain and sex (averaged from up to four technical replicates). Traits are colored on basis of overall glycosylation properties, i.e. overall complex type abundance (C, A2, A3, A3; black), high-mannose abundance/size and hybrid abundance (M, MM, Hy; green), fucosylation ( $F$; red), galactosylation (G; yellow), bisection (B; blue), acetylation (Ac; dark green), and sialylation (purple; various shades separating lactonization (L; $\alpha 2,3$-linkage), ethylation ( $\mathrm{E} ; \alpha 2,6$-linkage), total (S; all linkages) and branching sialylation (Sb; $\alpha 2,6-\mathrm{GlcNAc-linked)).} \mathrm{A)} \mathrm{Loading} \mathrm{plot} \mathrm{of} \mathrm{the}$ first two principal components (PC1 and PC2), respectively explaining $43 \%$ and $26 \%$ of the variance. B) Score plot of PC1 and PC2 colored by strain and sex. C) Score plot of PC1 and PC2 colored by sex. D) Score plot of PC1 and PC2 colored by strain. E) Loading plot of the third and fourth principal component (PC3 and PC4), respectively explaining $11 \%$ and $6 \%$ of the variance. F) Score plot of PC3 and PC4 colored by strain and sex. G) Score plot of PC3 and PC4 colored by sex. H) Score plot of PC3 and PC4 colored by strain. In general PC1 shows to largely separate the sexes, while PC2 separates strains BALB/c and C57BL/6 from strains CD-1 and Swiss Webster. The strains and sexes cluster well across the two components, but strains CD-1 and Swiss Webster show a larger individual variation than the BALB/C and CD57BL/6 strains. For a further listing of the principal components see Table $\mathbf{S 6 .}$ 
Figure S6. Overview of glycosylation traits (compositions and derived). Each point represents the technical average of one mouse individual, males and females respectively indicated by black and white coloring. A) Trait selection 1/6. For legends and calculations of the derived traits see Table $\mathbf{S 3}$.
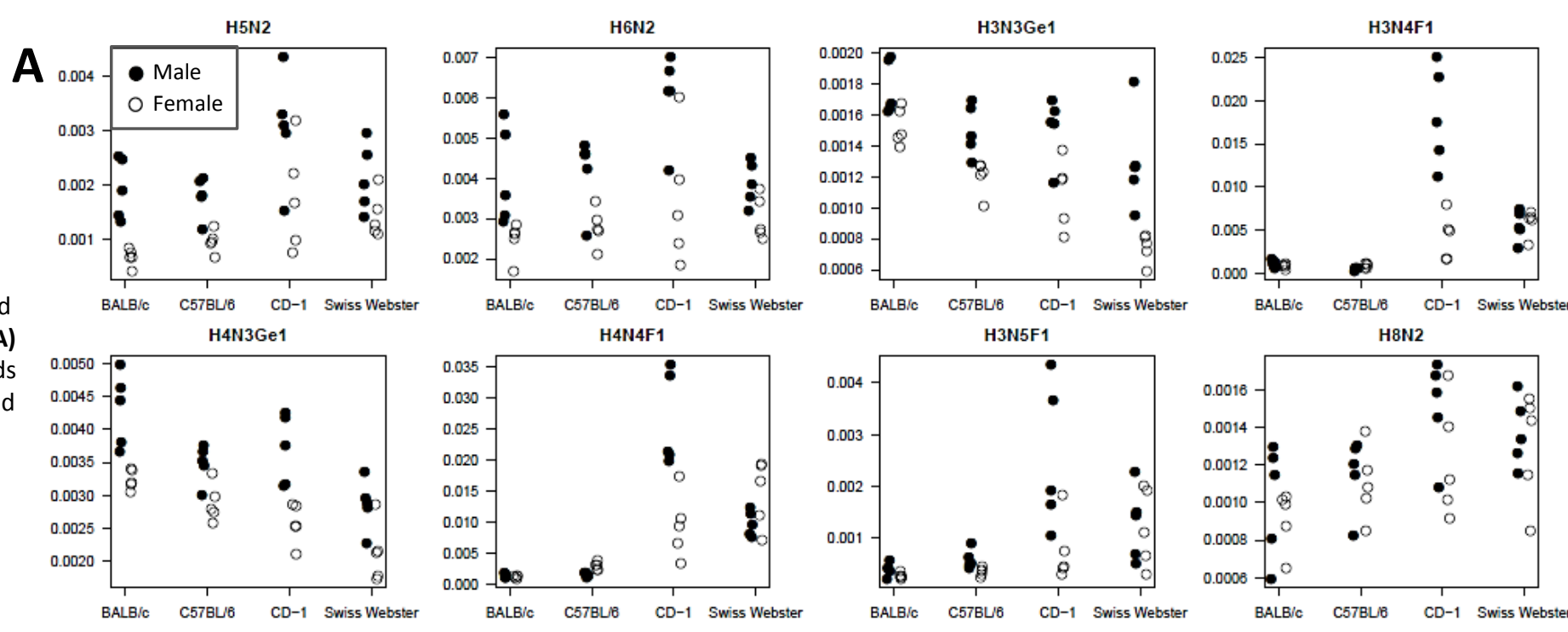

H4N4GI1
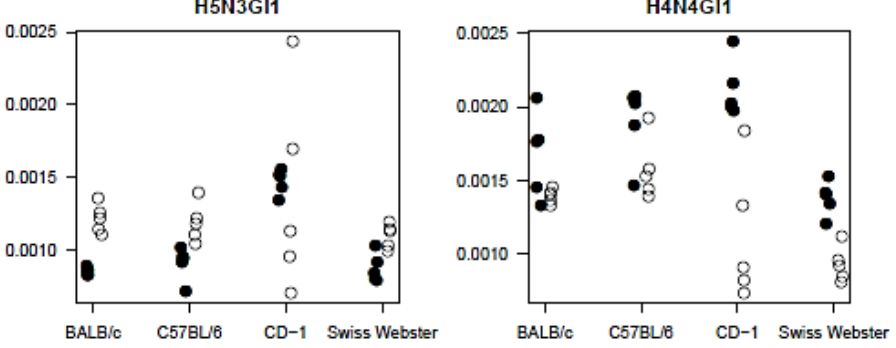

H5N3Ge1
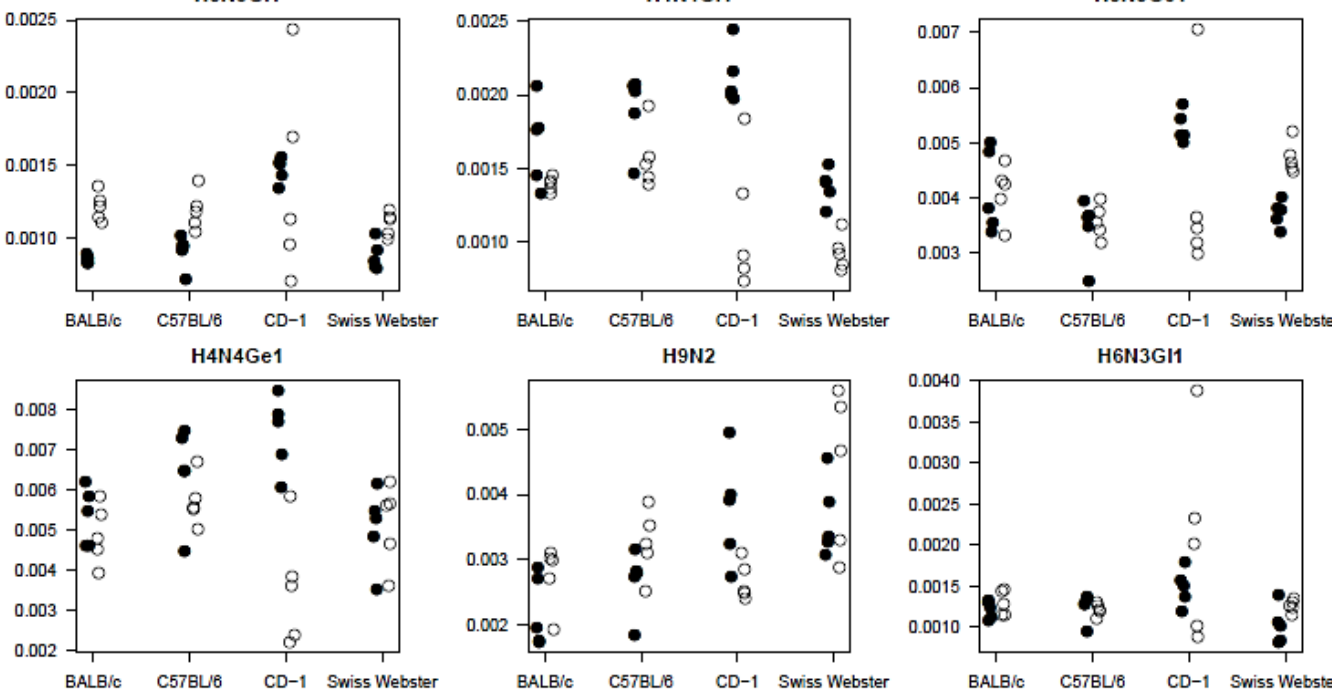

H6N3Ge1

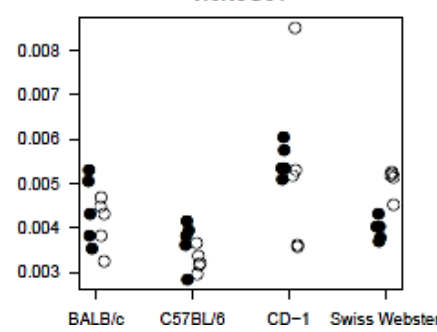

H9N2
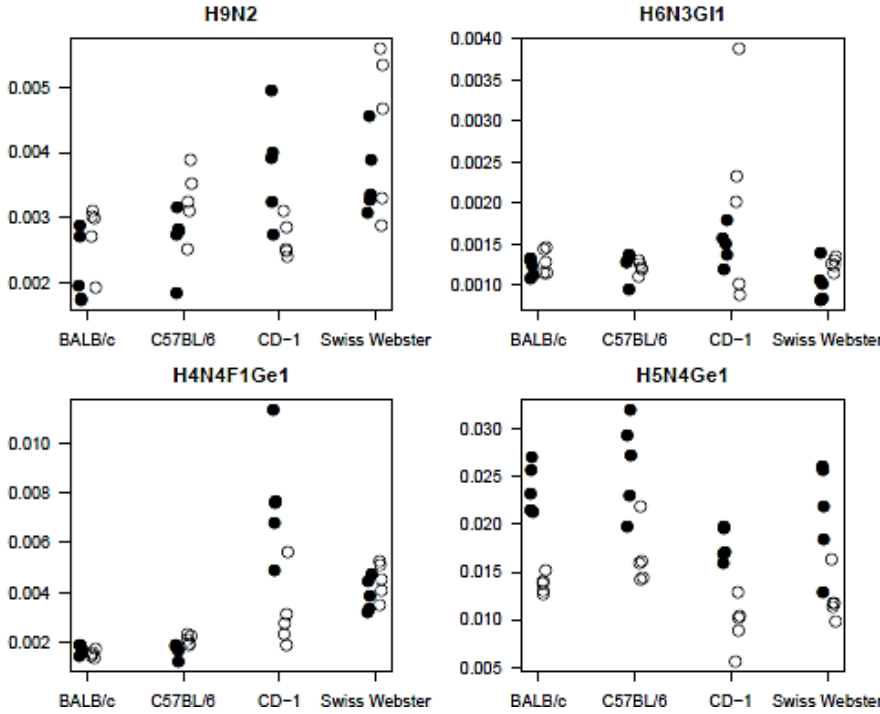

H5N4Ge1

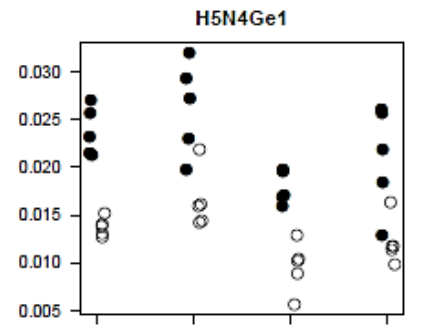

BALB/c C57BL6 CD-1 Swiss Webster
H5N4GI1

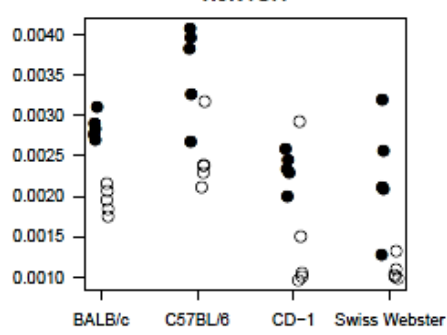

H5N4F1Ge1

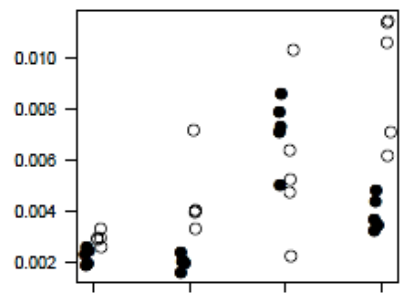

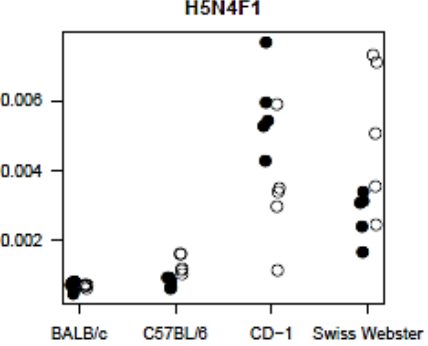


Figure S6. Overview of

glycosylation traits

(compositions and derived).

Each point represents the

technical average of one

mouse individual, males and

females respectively indicated

by black and white coloring. B)

Trait selection 2/6. For legends and calculations of the derived traits see Table $\mathbf{S 3}$.
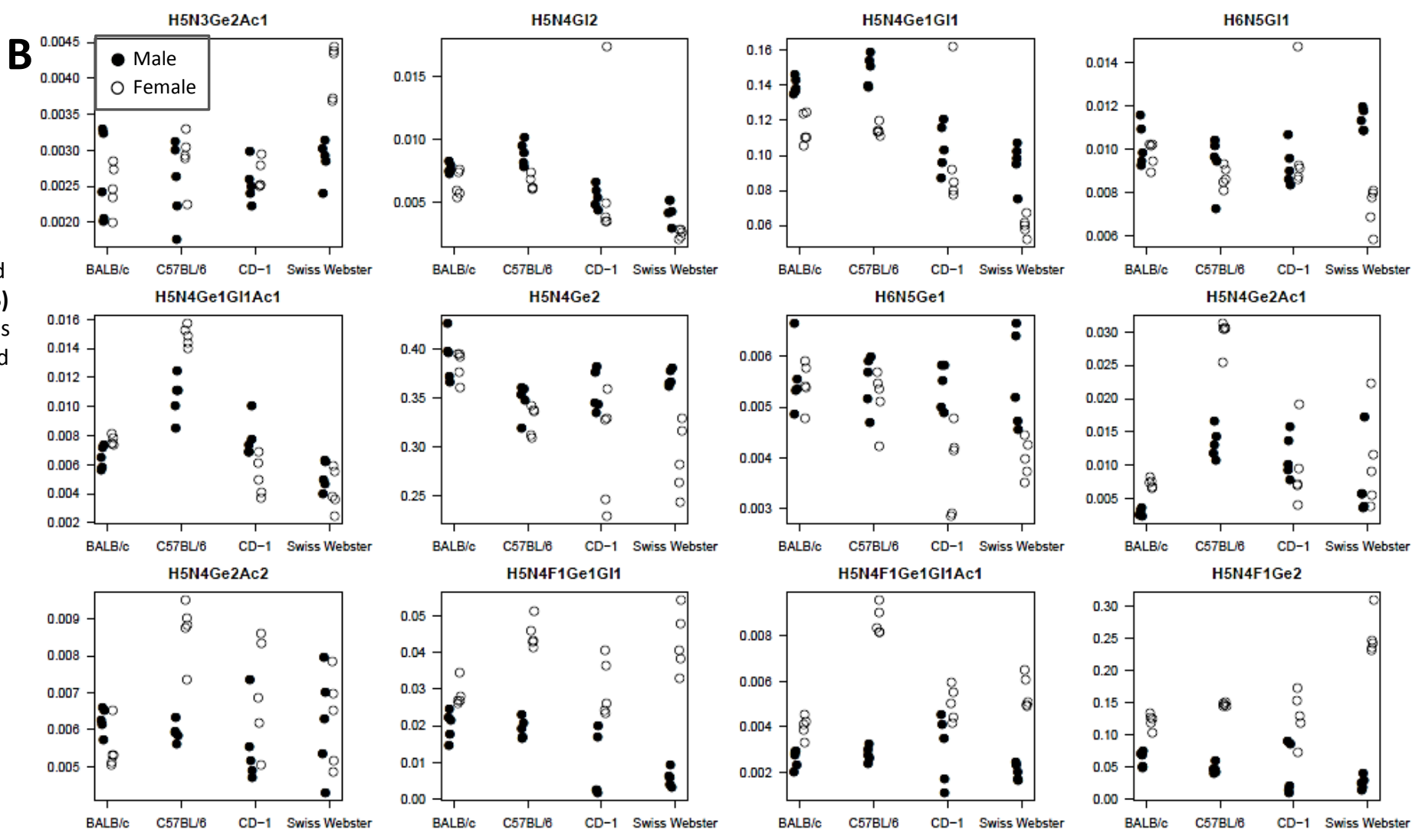

H5N4F1Ge2Ac2

H5N4Ge1GI2

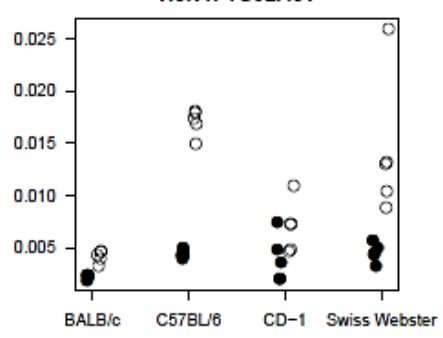

H6N5Ge1GI1

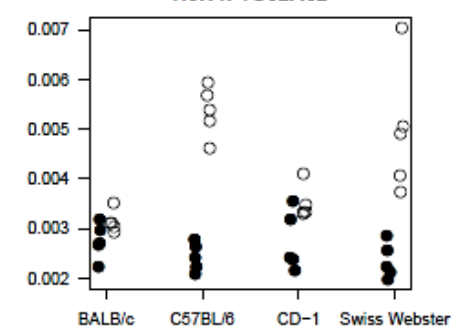

H5N4Ge2GI1Ac1
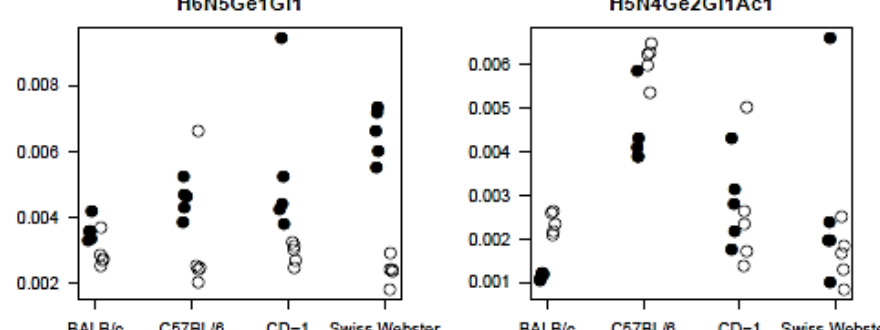

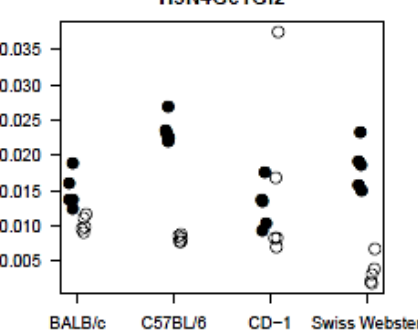

H5N4F1Ge1GI2

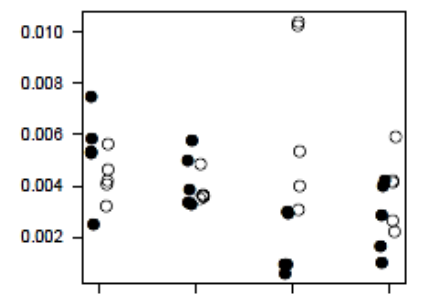

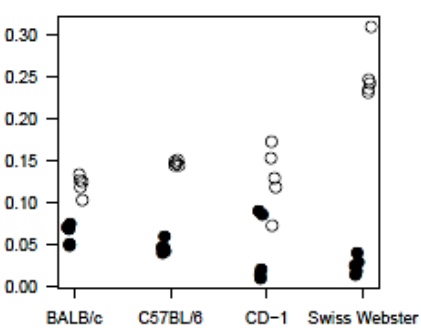

H5N4Ge2GI1

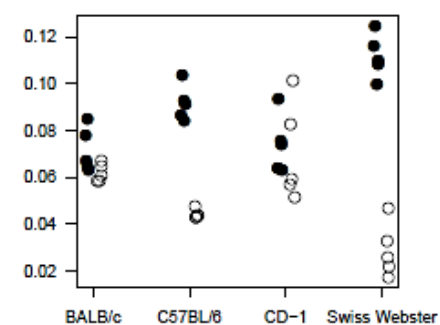

H5N4F1Ge2GI1

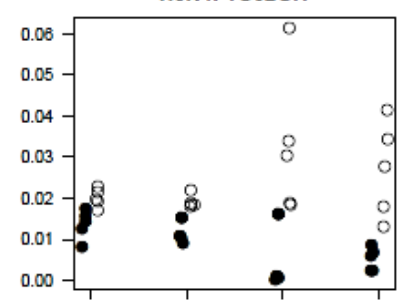

BALB/c C57BL/6 CD-1 Swiss Webs 
Figure S6. Overview of

glycosylation traits

(compositions and derived).

Each point represents the

technical average of one

mouse individual, males and

females respectively indicated

by black and white coloring. C)

Trait selection 3/6. For legends and calculations of the derived traits see Table $\mathbf{S 3}$.
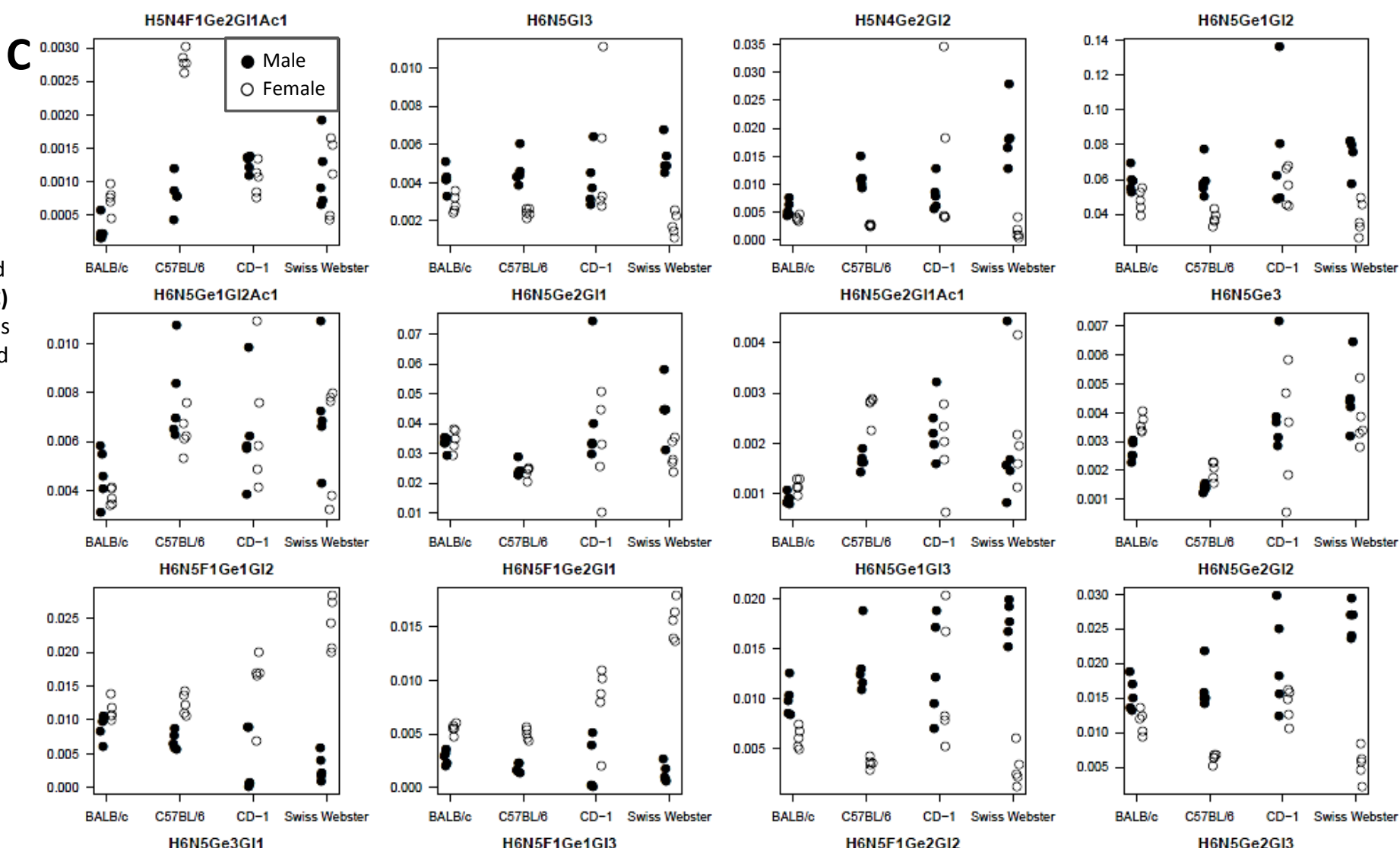

H6N5F1Ge2GI2

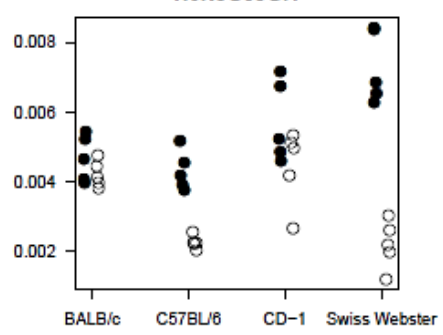

$\mathrm{M}$

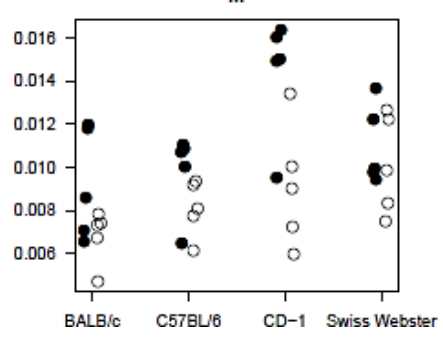

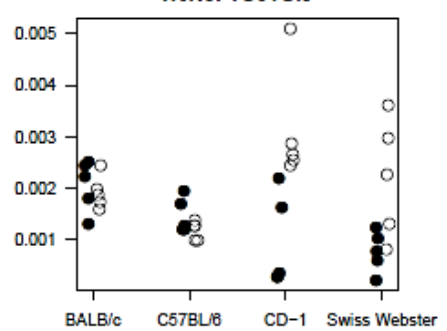

$\mathrm{Hy}$

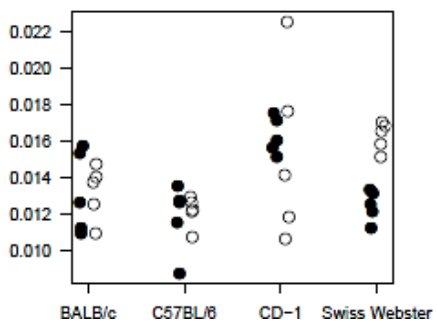

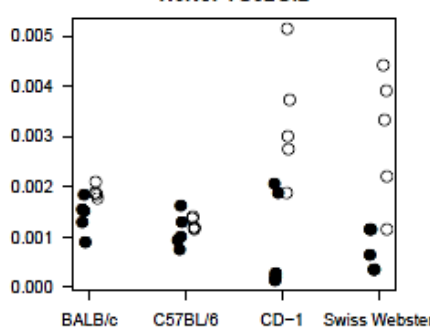

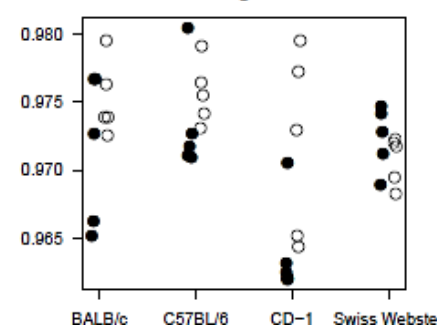

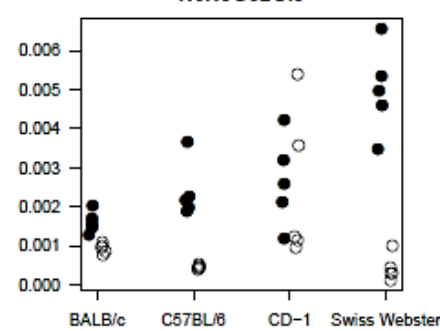

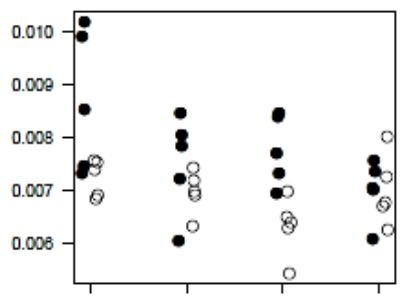


Figure S6. Overview of

glycosylation traits

(compositions and derived).

Each point represents the

technical average of one

mouse individual, males and

females respectively indicated

by black and white coloring. D)

Trait selection 4/6. For legends

and calculations of the derived

traits see Table $\mathbf{S 3}$.

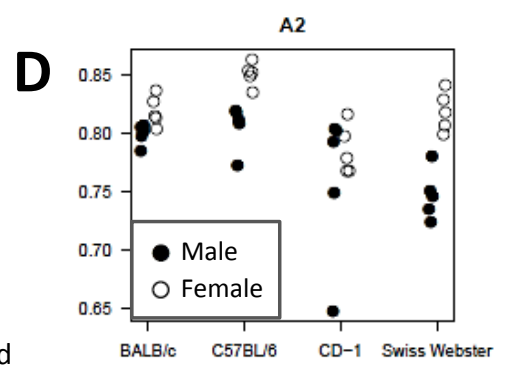

MM

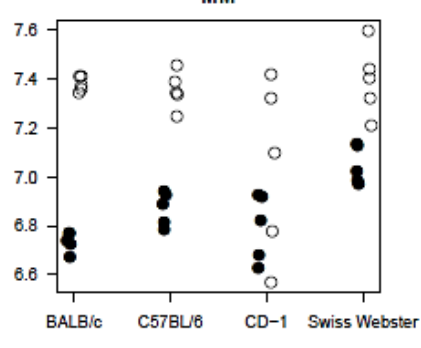

A2FB
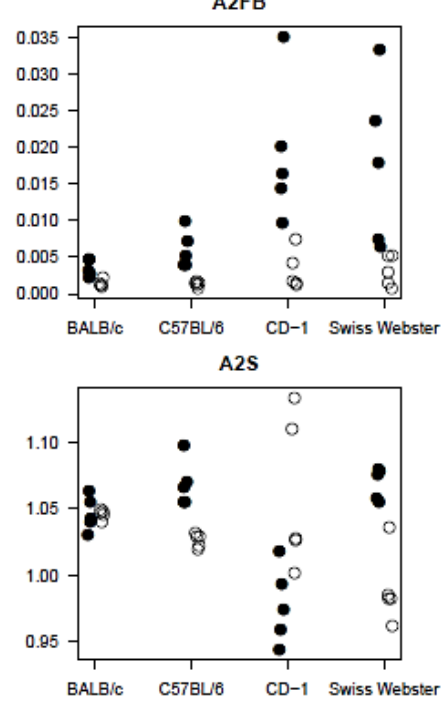

A2FS

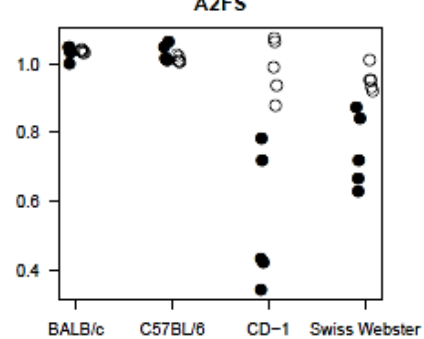

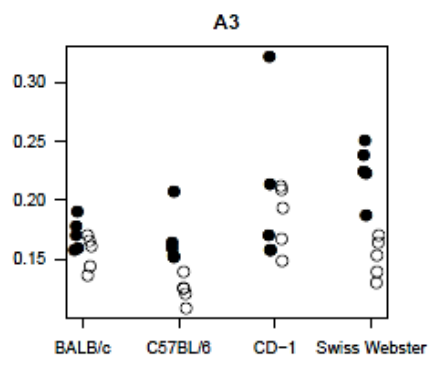

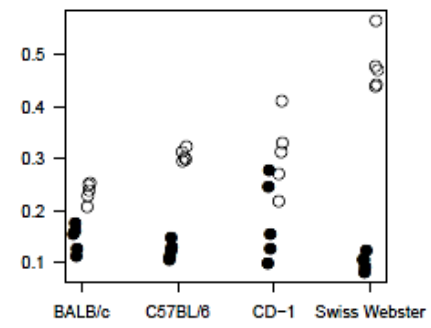

A2G

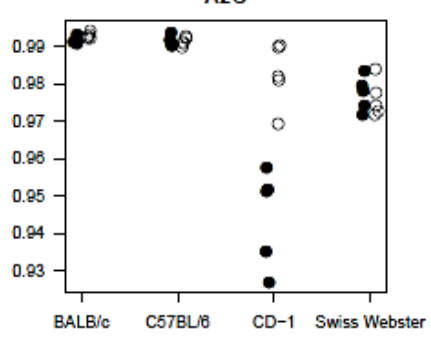

A3S

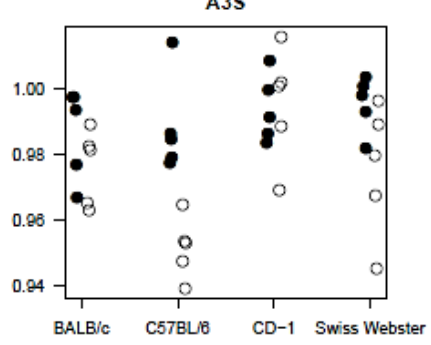

A3FS

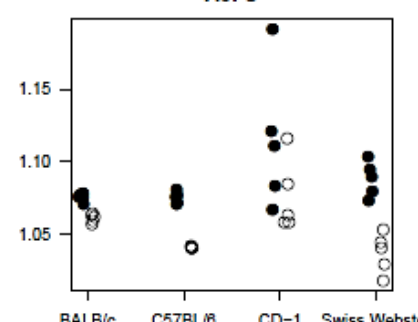

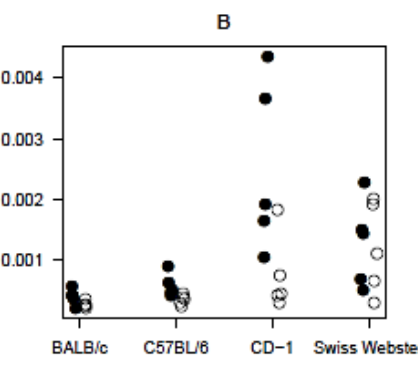

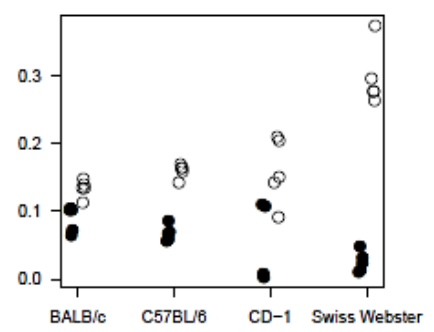

A2F0G

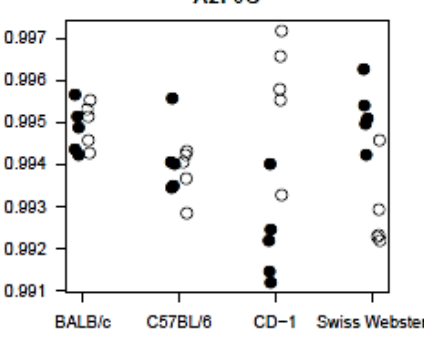

A2F0S
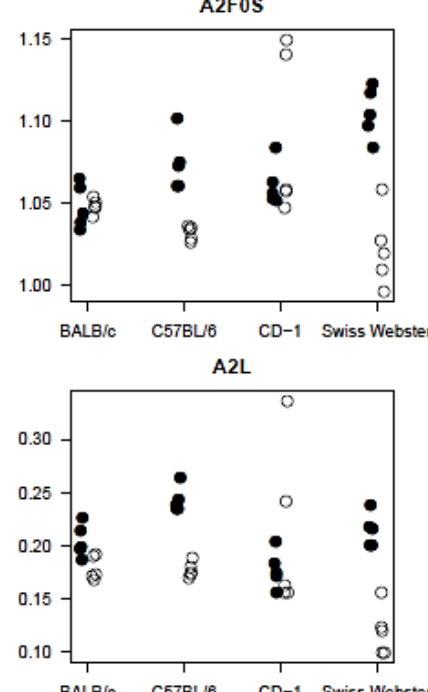
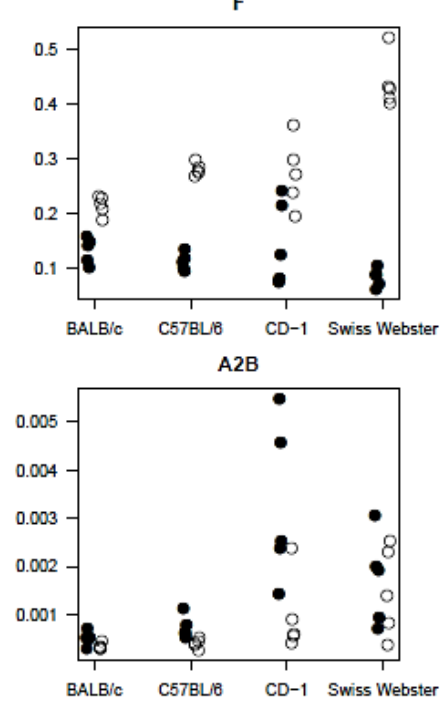

A2FG
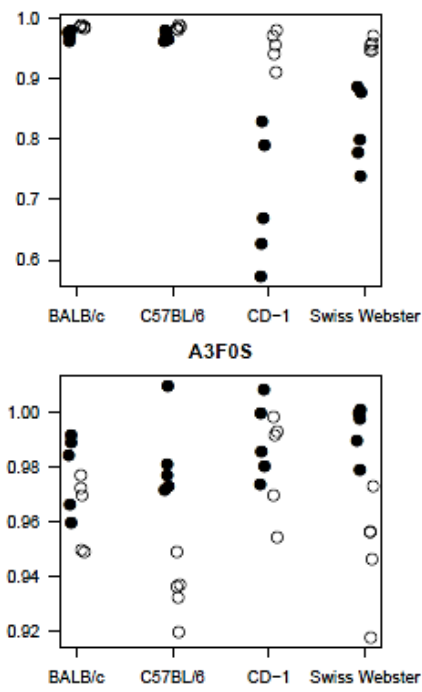

A3L

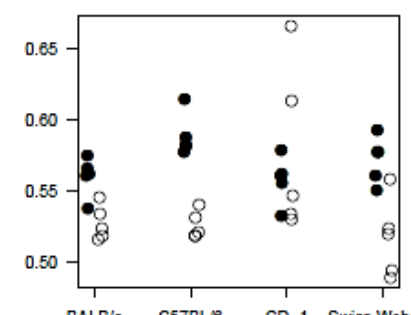


Figure S6. Overview of

glycosylation traits

(compositions and derived).

Each point represents the

technical average of one

mouse individual, males and

females respectively indicated

by black and white coloring. E)

Trait selection 5/6. For legends and calculations of the derived traits see Table $\mathbf{S 3}$.
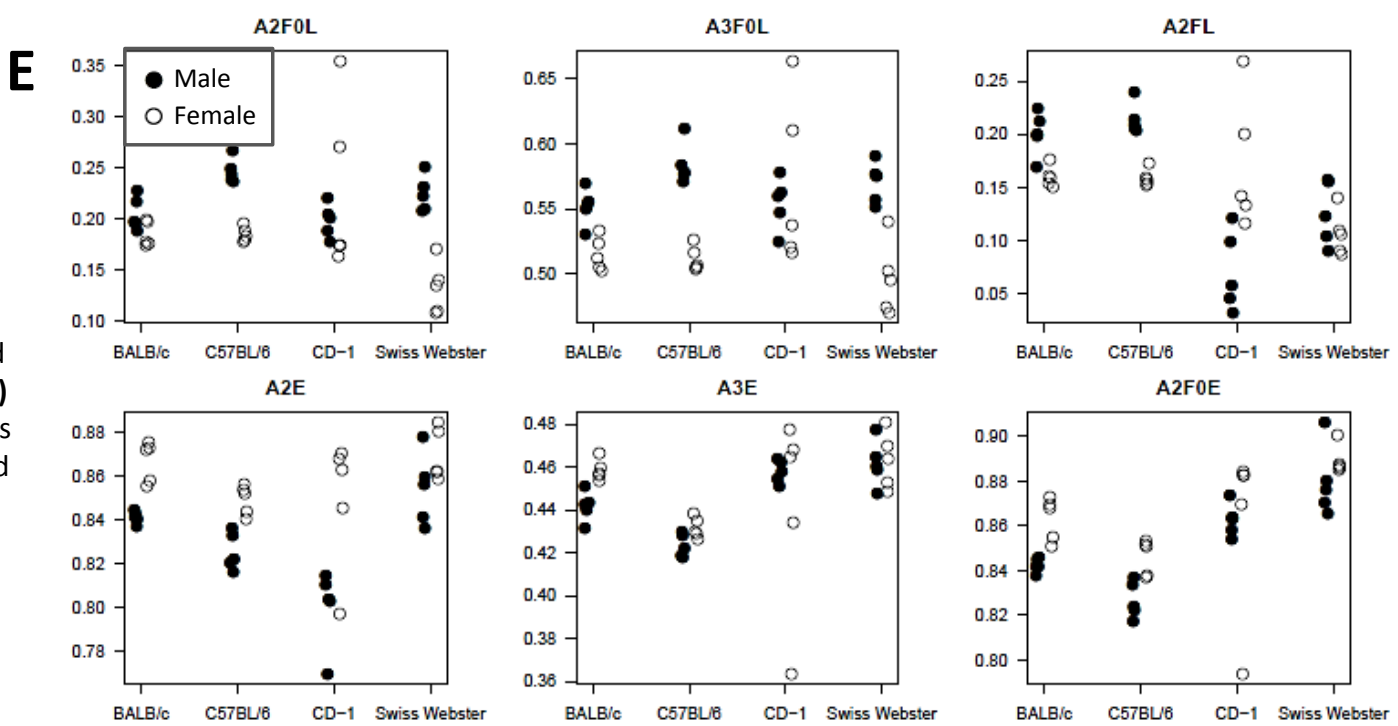

A2FE

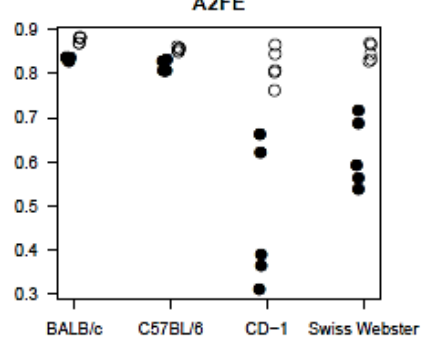

A2F0GS

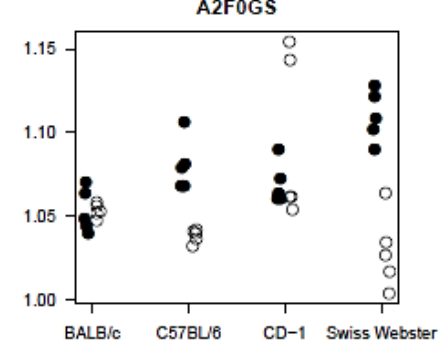

A2GL

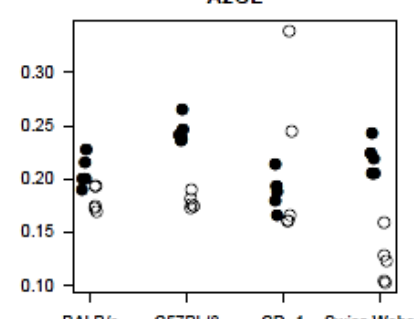

A3E

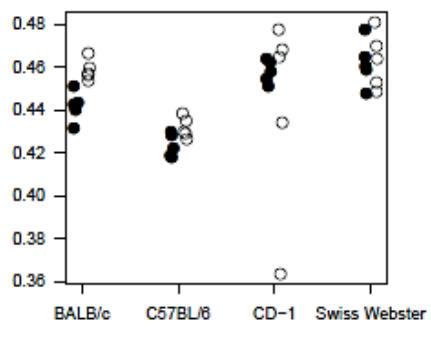

A3FE

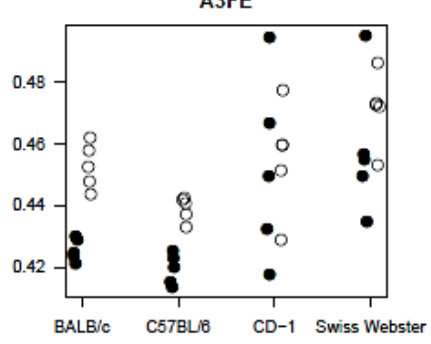

A3F0GS

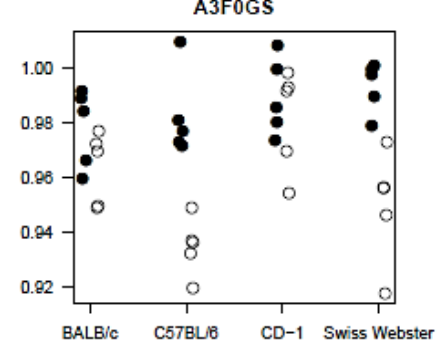

A3GL

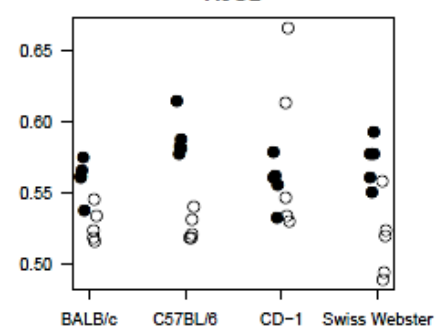

A2F0E

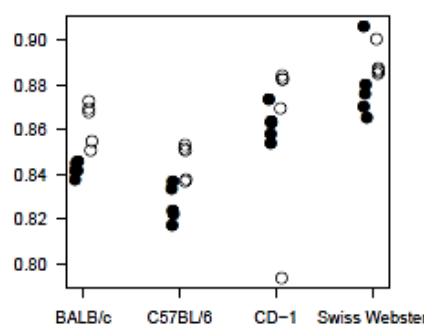

A2GS

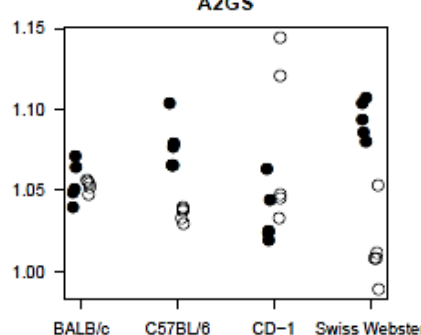

A2FGS

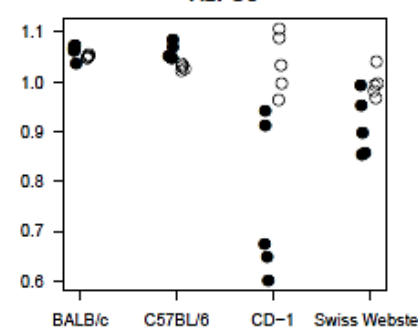

A2F0GL

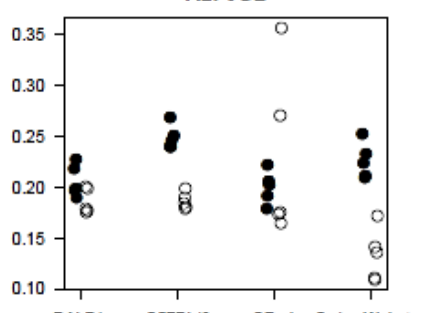

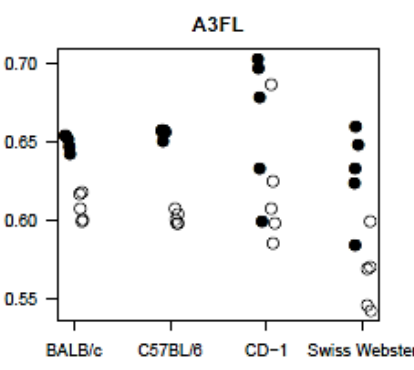

A3F 0 E

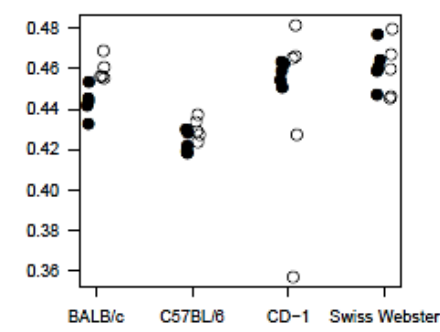

A3GS

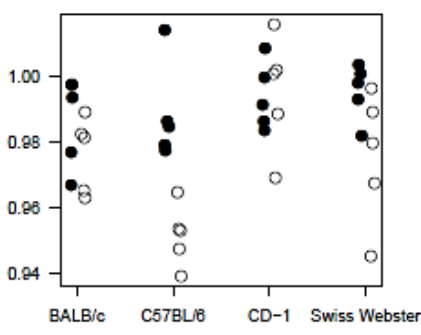

A3FGS

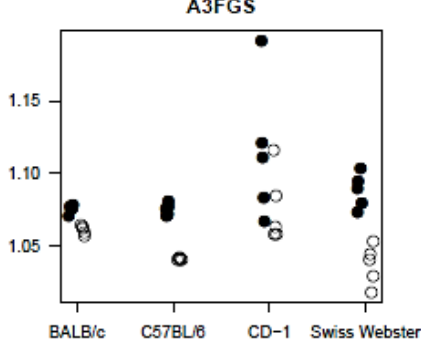

A3F0GL

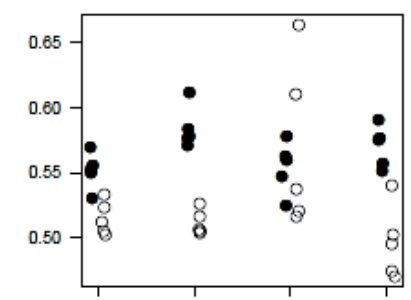


Figure S6. Overview of

glycosylation traits

(compositions and derived).

Each point represents the

technical average of one

mouse individual, males and

females respectively indicated

by black and white coloring. F)

Trait selection 6/6. For legends and calculations of the derived traits see Table $\mathbf{S 3}$.
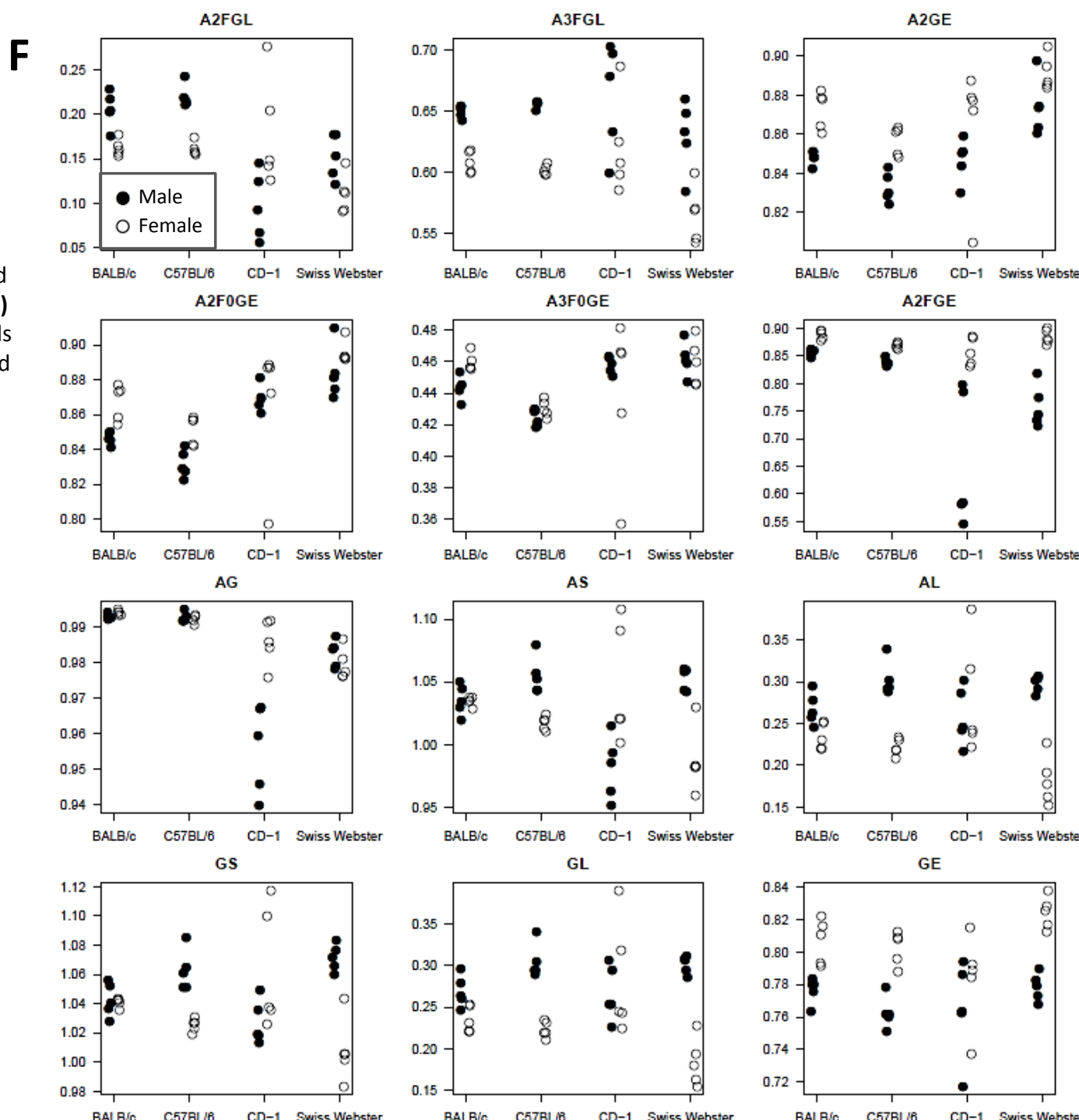

A2S

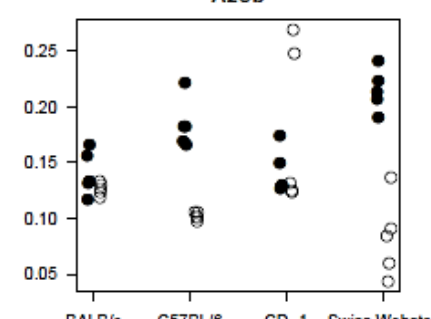

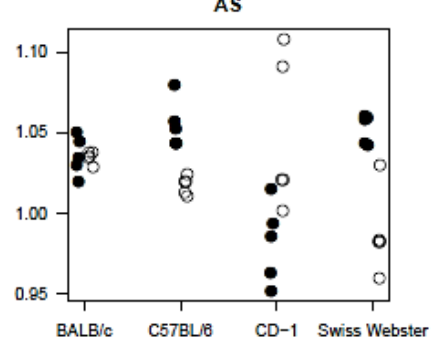

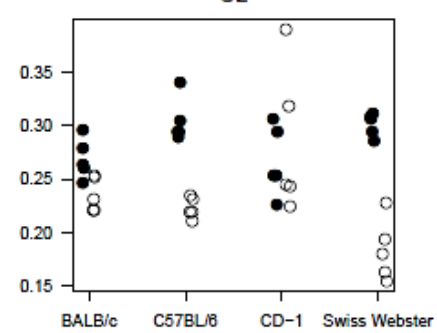

A3Sb

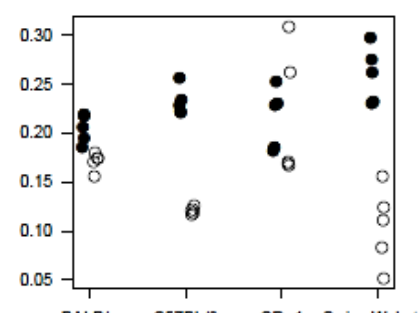

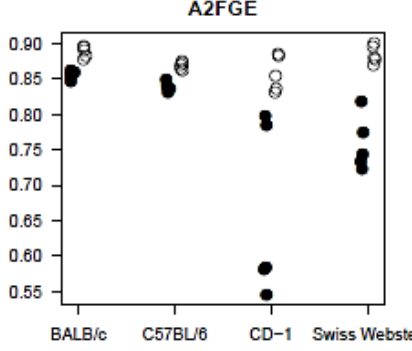

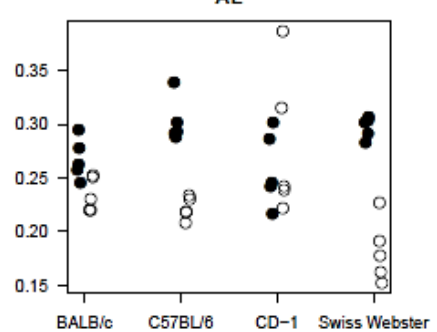

GE

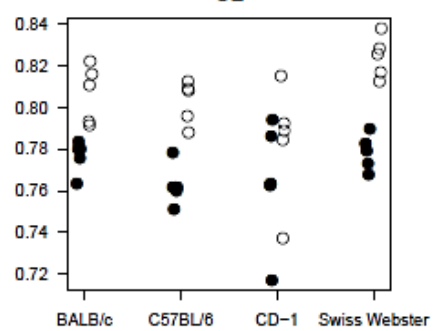

sb

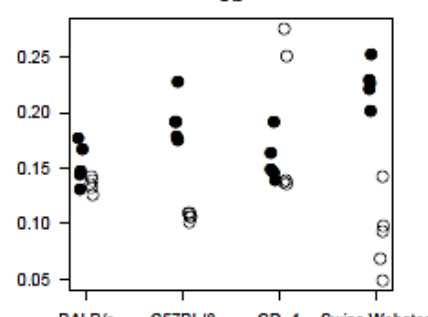

A3GE

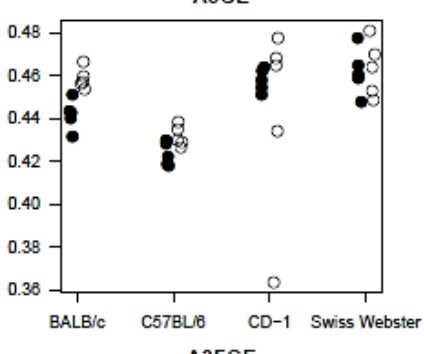

A3FGE
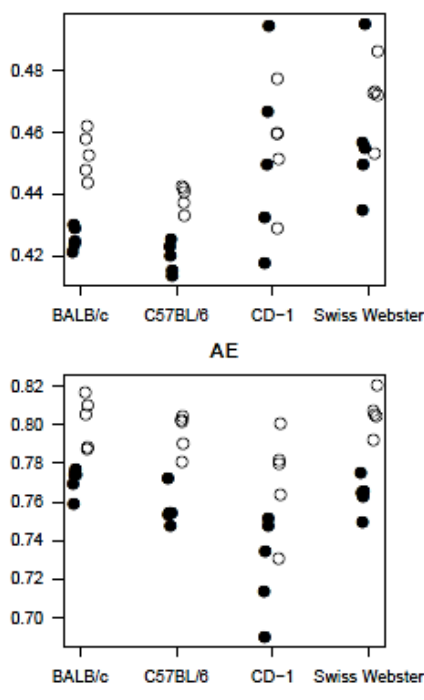

$\mathrm{A} 1 \mathrm{Sb}$

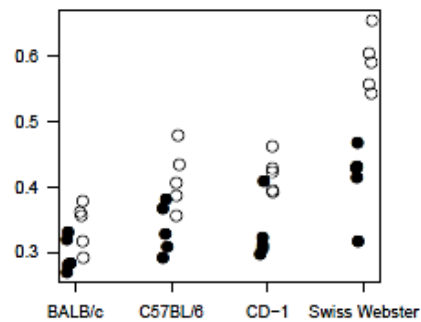

SAC

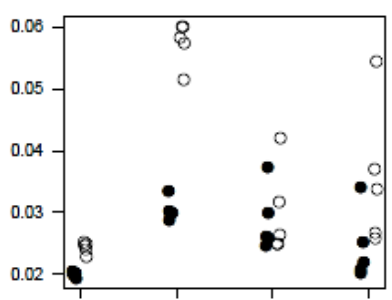

BALB/c C57BL/6 CD-1 Swiss Webs

Page S-21 


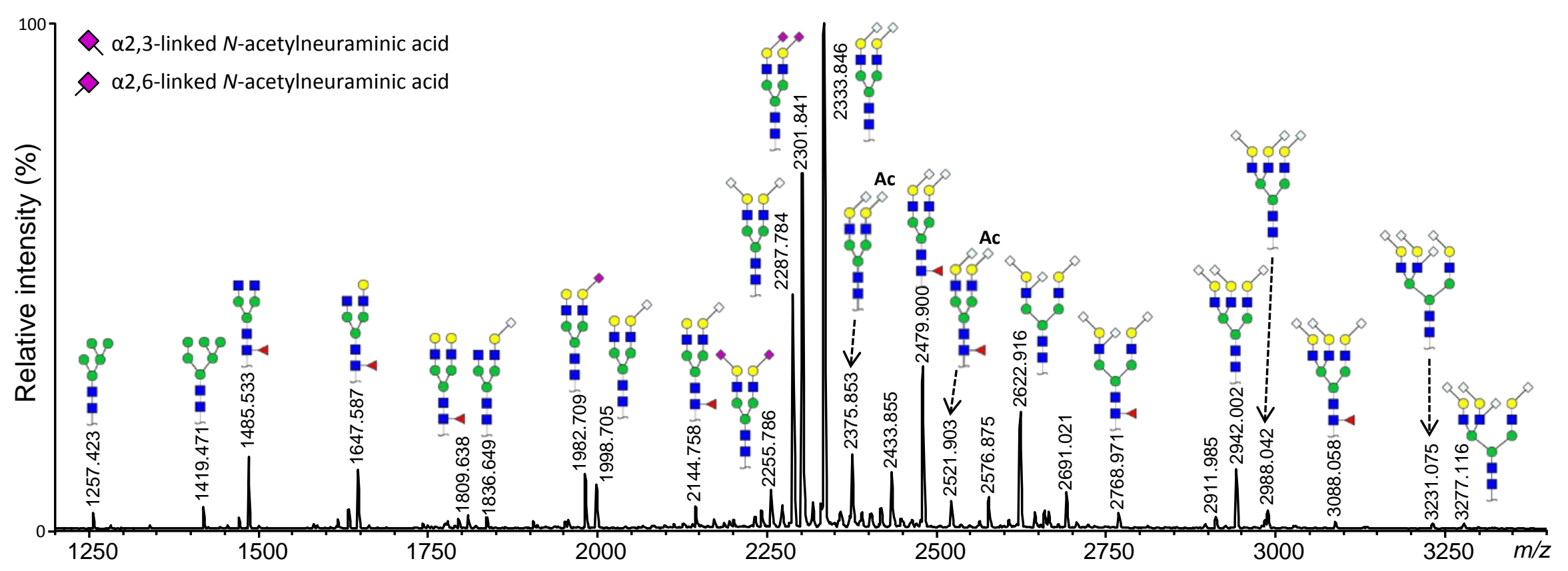

Supporting Figure S7. Mixed sex plasma N-glycome profile of C57BL/6 mice as measured by reflectron positive mode MALDI-TOF-MS after ethyl esterification. Glycan species are considered $[\mathrm{M}+\mathrm{Na}]^{+}$and assigned to signals on basis of recorded $m / z$ values and fragmentation. In addition to $N$-glycolylneuraminic acid, this sample shows the presence of $\mathrm{N}$-acetylneuraminic acid, thereby demonstrating the separate detectability of the sialic acid types. 